\title{
ANÁLISE DAS AÇÕES JUDICIAIS INDIVIDUAIS PARA FORNECIMENTO DE MEDICAMENTOS EM SÃO JOÃO DA BOA VISTA
}

Analysis of individual lawsuits on supplying medication in São João da Boa Vista, São Paulo, Brazil

${ }^{1}$ Centro Universitário das Faculdades Associadas de Ensino. São João da Boa Vista/SP, Brasil.

${ }^{2}$ Universidade Estadual Paulista “Júlio de Mesquita Filho”. São Paulo/SP, Brasil. Correspondência: Ildelisa Cabral. E-mail: ildelisa@adv.oabsp.org.br.

Recebido em: 04/08/2014. Revisado em: 13/11/2014. Aprovado em: 17/11/2014. 


\section{RESUMO}

O presente artigo tem por objetivo analisar as ações judiciais individuais realizadas para garantir o acesso a medicamentos no município de São João da Boa Vista (SP). Tratou-se de um estudo longitudinal retrospectivo, de caráter exploratório documental, nos anos de 2009 a 2012, tendo como objeto de análise processo judicial movido individualmente em face do Município com a finalidade de fornecimento de medicamento. Após a análise de 87 ações judiciais, foram encontrados os seguintes resultados: mandado de segurança individual interposto por mulheres beneficiárias da gratuidade de justiça, com patrocínio de advogados particulares, em que a ré é a diretora do Departamento Municipal de Saúde, solicitando, através de receitas privadas, medicamentos pelo nome comercial, não listados pelo Sistema Único de Saúde, todos com registro na Agência Nacional de Vigilância Sanitária. Os resultados obtidos são compatíveis com os encontrados pela literatura. Concluiu-se que, a fim de garantir o direito constitucional à saúde dos indivíduos, será necessária uma melhor organização do Estado quanto à assistência farmacêutica.

\section{Palavras-Chave}

Ações judiciais; Judicialização; Medicamentos; Sistema Único de Saúde.

\section{ABSTRACT}

This study seeks to analyze the individual lawsuits filed to guarantee access to medication in the city of São João da Boa Vista, São Paulo State, Brazil. It is a longitudinal retrospective study that examines documents from 2009 to 2012 to analyze the lawsuits filed by individuals against the city government to receive supplies of medication. A total of 87 lawsuits were analyzed, and the following results were found: individual injunctions filed by women beneficiaries of the gratuity of righteousness, with sponsorship from private lawyers, in which the defendant is the director of the Municipal Department of Health. Through private prescriptions, the women solicited name-brand medications not listed by the Brazilian Public Health System (SUS), all of which are registered with the Brazilian National Health Surveillance Agency (Anvisa). The results obtained are compatible with those found in the literature. It can be concluded that, to guarantee individuals' constitutional right to health it is necessary a better organization of the State regarding pharmaceutical assistance.

\section{Keywords}

Brazilian Public Health System; Lawsuits; Legalization; Medications. 


\section{Introdução}

Desde a Constituição Federal de $1988(\mathrm{CF} / 88)^{1}$ e a Lei n. 8.080/1990 (Lei Orgânica da Saúde $)^{2}$, são recorrentes as ações judiciais interpostas por pessoas doentes que necessitam de medicamentos de todos os tipos ${ }^{3,4,5}$.

O direito à assistência farmacêutica é parte integrante do direito constitucional à saúde - sendo este um dos direitos sociais - e constitui-se de prestações positivas proporcionadas pelo Estado, direta ou indiretamente, enunciadas em normas constitucionais, que possibilitam melhores condições de vida aos mais fracos. Esses direitos tendem a equalizar situações sociais desiguais ${ }^{6}$.

Em razão do disposto constitucionalmente, entrou em vigor a Lei Orgânica da Saúde, estabelecendo a estrutura e o modelo operacional do Sistema Único de Saúde (SUS), concebido como o conjunto de ações e serviços de saúde prestados por órgãos e instituições públicas federais, estaduais e municipais, da administração direta e indireta ${ }^{7}$.

A saúde é uma área de grande preocupação, tanto para os indivíduos quanto para o governo: os primeiros reclamam e solicitam maiores investimentos públicos no setor; o governo, por sua vez, investe parcela considerável do orçamento na saúde e, ainda assim, não consegue satisfazer essa necessidade básica elementar.

Para Vieira, "pode-se argumentar que os recursos da saúde não são suficientes e que é preciso aumentar o aporte financeiro para o setor. Sobre isso não há dúvidas. Entretanto, há sempre um limite"».

\footnotetext{
${ }^{1}$ BRASIL. Constituição da República Federativa do Brasil. Disponível em: <http://www.planalto.gov.br/ccivil_03/Constituicao/Constituicao.htm>. Acesso em: 23 out. 2011.

${ }^{2}$ BRASIL. Lei n. 8.080 de 19 de setembro de 1990. Dispõe sobre as condições para a promoção, proteção e recuperação da saúde, a organização e o funcionamento dos serviços correspondentes e dá outras providências. Disponível em: <http://www.planalto.gov.br/ccivil_03/leis/L8080.htm>. Acesso em: 23 out. 2011.

${ }^{3}$ MESSEDER, A. M.; CASTRO, C. G. S. O. de; LUIZA, V. L. Mandados judiciais como ferramenta para garantia do acesso a medicamentos no setor público: a experiência do Estado do Rio de Janeiro, Brasil. Cad. Saúde Pública, Rio de Janeiro, v. 21, n. 2, p. 525-534, mar./abr. 2005. http://dx.doi.org/10.1590/ S0102-311X2005000200019.

${ }^{4}$ BRANCO, L. T. C. Abrangência do direito à saúde: fornecimento de medicamentos especiais é dever do Estado? Revista do Movimento do Ministério Público Democrático, São Paulo, ano 4, n. 13, maio 2007.

${ }^{5}$ SANT'ANA, J. M. B. Essencialidade e assistência farmacêutica: um estudo exploratório das demandas judiciais individuais para acesso a medicamentos no Estado do Rio de Janeiro. 2009. 94p. Dissertação (Mestrado) - Escola Nacional de Saúde Pública Sergio Arouca, ENSP, Rio de Janeiro, 2009.

${ }^{6}$ MARQUES, S. B.; DALLARI, S. G. Garantia do direito social à assistência farmacêutica no Estado de São Paulo. Revista de Saúde Pública, São Paulo, v. 41, n. 1, p. 101-107, fev. 2007. http://dx.doi.org/10.1590/S0034-89102007000100014.

${ }^{7}$ BARROSO, L. R. Da falta de efetividade à judicialização excessiva: direito à saúde, fornecimento gratuito de medicamentos e parâmetros para a atuação judicial. Rio de Janeiro, 2007. Disponível em: <http://s.conjur.com.br/dl/estudobarroso.pdf>. Acesso em: 22 out. 2011.

${ }^{8}$ VIEIRA, F.S. Ações judiciais e direitoà saúde: reflexão sobrea observância aos princípios doSUS. Revista de Saúde Pública. São Paulo, v. 42, n. 2, 367, fev. 2008. http://dx.doi.org/10.1590/S0034-89102008005000010.
} 
As pesquisas existentes abordando essa temática tratam de demandas judiciais em estados ou em cidades de grande porte, não tendo sido encontrado estudo elaborado em cidade do mesmo porte de São João da Boa Vista.

O Município de São João da Boa Vista, segundo o Censo Demográfico 2010, possui 83.639 habitantes e é considerado, no indicador saúde, como de alto desenvolvimento ${ }^{9,10}$.

O objetivo do presente trabalho foi analisar as ações judiciais individuais para garantir o acesso a medicamentos em São João da Boa Vista.

\section{Caracterização dos processos judiciais}

A unidade de análise de estudo foram cópias de todos os processos judiciais referentes às ações movidas por indivíduos em face do Município de São João da Boa Vista ou em face da diretora do Departamento Municipal de Saúde, que tinham por objeto o fornecimento de medicamentos, no período de 2009 a 2012. Essas cópias de processos judiciais se encontravam na Assessoria Jurídica Municipal.

Fez-se a opção pelo período de quatro anos (2009 a 2012) para coincidir com um mandato de governo municipal, bem como pela existência de registro dos dados pela Prefeitura Municipal e também para se obter representatividade nos dados encontrados.

O critério de inclusão foi o seguinte: todas as ações judiciais individuais pleiteando fornecimento de medicamentos em que o réu fosse a diretora do Departamento Municipal de Saúde ou o Município de São João da Boa Vista, interpostas no período de 2009 a 2012, com ou sem sentença.

A fonte de dados primária foi a Assessoria Jurídica Municipal. Realizou-se um levantamento, por meio do sistema informatizado, por uma funcionária do órgão, a fim de se listarem todos os processos judiciais arquivados e em andamento. Depois disso, foram identificadas manualmente as ações que se enquadravam nos critérios de inclusão. Assim, selecionados os processos, a própria pesquisadora realizou consulta às cópias das peças processuais mais relevantes; as ações em andamento foram fotografadas no local, e as ações já arquivadas foram xerocopiadas.

A segunda fonte de dados foi obtida pela pesquisadora no Setor de Planejamento e Controle Orçamentário, que é a Assessoria Técnica do Departamento de Finanças Municipal, através da consulta ao orçamento do Departamento Municipal de Saúde, cujos dados foram fotografados no local.

9IBGE. Cidades@. Disponível em: <http://cidades.ibge.gov.br/painel/painel.php?codmun=354910>. Acesso em: 16 fev. 2014.

${ }^{10}$ FIRJAN. Índice FIRJAN de desenvolvimento municipal. Disponível em: <http://www.firjan.org.br/ifdm/ consulta-ao-indice/consulta-ao-indice-grafico.htm?UF=SP\&IdCidade=354910\&Indicator-4\&Ano=2010>. Acesso em 16 jan. 2014. 
A terceira fonte de dados foi obtida pela pesquisadora no Departamento Municipal de Saúde, através da consulta de planilha já elaborada por este órgão, demonstrando as despesas com assistência farmacêutica, cujos dados foram fotografados no local.

Os dados foram coletados por meio de instrumento semiestruturado, contendo 32 questões fechadas, tendo sido uma adaptação do instrumento utilizado por Sant'Ana ${ }^{11}$, em sua dissertação de mestrado.

A verificação do registro na Agência Nacional de Vigilância Sanitária (Anvisa) levou em consideração o medicamento solicitado. Teve como fonte o respectivo sítio da agência reguladora na internet (http://www.anvisa.gov.br).

A verificação da lista de medicamentos de fornecimento obrigatório pelo município e pelo estado teve como fonte o sítio da saúde na internet (http://portalsaude.saude.gov.br).

Como a maioria dos medicamentos solicitados estava descrita por seus nomes comerciais e a lista oficial do SUS apresenta os medicamentos por sua denominação genérica, procedeu-se à obtenção da composição do produto por meio do endereço eletrônico (http://www.bulas.med.br).

Este trabalho sofreu uma limitação com relação ao ano de 2009, no tocante à informação obtida junto ao Departamento Municipal de Saúde quanto aos gastos efetivos com as ações judiciais. Isso se deu pelo fato de que, naquele ano, o município procedeu a uma modificação em seu sistema operacional, em todos os departamentos, o que impossibilitou o acesso ao valor despendido - ou seja, só há informação a partir do ano de 2010.

\section{Resultados}

No período de 2009 a 2012 foram identificadas, na listagem da Assessoria Jurídica do Município de São João da Boa Vista, 96 ações solicitando medicamentos, sendo que 18 delas já estavam arquivadas e 78 estavam em andamento.

De todas as 18 ações arquivadas foram encontradas suas respectivas cópias para consulta.

Das 78 ações em andamento, sete não foram encontradas no respectivo órgão municipal e duas foram excluídas por não se encaixarem nos critérios de inclusão da pesquisa, o que resultou em 69 ações em andamento.

Chegou-se, portanto, a 18 ações arquivadas e a 69 ações em andamento. Desta forma, o universo da pesquisa foi de 87 cópias de ações solicitando medicamentos.

\footnotetext{
${ }^{11}$ SANT'ANA, J. M. B. op. cit.
} 
Em todas as 87 ações, a natureza do autor foi particular, ou seja, nenhuma delas foi ingressada por associação de pacientes ou pelo Ministério Público.

A maioria das ações foi interposta por mulheres: 56 ações, representando 64,37\%.

Quanto à classificação da ação escolhida, há a predominância de mandados de segurança: 74 ações, representando 85,06\%.

Com relação à representação jurídica, há o predomínio de advogados particulares, em 49 das ações, representando 56,32\%, sendo que as outras 38 ações foram patrocinadas por meio de um convênio entre a Ordem dos Advogados do Brasil e a Procuradoria Geral do Estado. (OAB/PGE).

A gratuidade de justiça foi concedida em 82 ações, representando 94,25\%, sendo que somente em uma ação não houve a concessão e em quatro ações não foi possível encontrar essa informação.

A diretora do Departamento Municipal de Saúde foi ré em 49 ações, representando 56,32\%, seguida da diretora do Departamento Municipal de Saúde e do diretor do Departamento Regional de Saúde XIV em conjunto, em 19 ações, significando 21,24\%.

No que se refere ao resultado das sentenças, a maioria das ações foi julgada procedente: 81 delas, representando $93,10 \%$.

A receita médica proveniente do serviço privado foi maioria, pois originou 61 ações, o que representou 70,11\%. Apesar de a maioria das ações solicitar um único medicamento, foi comum a solicitação de vários medicamentos em uma única ação, chegando até mesmo a nove remédios pedidos. No total das 87 ações pesquisadas, foram solicitados 227 medicamentos, uma média de 2,61 medicamentos solicitados por ação judicial.

Com relação à competência para fornecimento dos medicamentos requeridos judicialmente, 56,39\% não pertenciam à lista oficial; 30,40\% eram de responsabilidade do município; e 13,21\% eram de responsabilidade do estado - em um total de 227 medicamentos solicitados. Todos os medicamentos possuíam registro na Anvisa.

Quanto à primeira compra de medicamentos, a fim de atender à ordem judicial de entrega em um prazo exíguo de dias, se o Departamento Municipal de Saúde não tivesse o medicamento solicitado judicialmente, o Departamento de Compras do Município de São João da Boa Vista fez uma cotação, pelo menor preço, em três farmácias locais: Droganova, Drogaria Familiar e Farmácia do Povo, em razão de todas emitirem nota fiscal eletrônica. As demais compras de medicamentos, para continuar atendendo à ordem judicial, foram feitas por meio de licitação.

A representatividade dos gastos com ações judiciais em relação às despesas com assistência farmacêutica, ano a ano entre 2010 e 2012, quase dobrou. Ou seja, em 2010 esses gastos representavam 18,76\%, ao passo que em 2012 representaram $30,20 \%$. 
O gasto médio por ação judicial foi de R \$ 5.994,95 em 2010; ao passo que, em 2012 o gasto médio por ação foi de $\mathrm{R} \$ 25.577,80$.

\section{Discussão}

\section{Natureza do autor da ação}

Todos os autores das 87 ações pesquisadas em São João da Boa Vista ingressaram individualmente para solicitar os medicamentos, dado também encontrado por Marques e Dallari ${ }^{12}$ no Estado de São Paulo, e por Silva et al. ${ }^{13}$ no Estado do Espírito Santo.

Travassos et al. ${ }^{14}$ identificaram, nos Tribunais de Justiça dos estados de Minas Gerais, Rio Grande do Sul e Pernambuco, autores individuais como a maioria dos titulares das ações; Romero ${ }^{15}$, no Distrito Federal, encontrou 95\% de autores particulares conduzidos pela Defensoria Pública.

Com essas demandas judiciais, pretende-se garantir direitos sociais que são essencialmente coletivos. Entretanto, como a atuação do Poder Judiciário ocorre no âmbito individual, [...] acaba havendo tratamento diferenciado, privilegiando esses pacientes, o que é incompatível com a ideia de igualdade proposta pelo SUS ${ }^{16}$.

\section{Classificação da ação}

O mandado de segurança foi a ação mais escolhida no Município de São João da Boa Vista, resultado verificado também no Tribunal de Justiça do Estado de Pernambuco por Travassos et al. ${ }^{17}$. Essa preferência pode ser explicada pelo fato de que não se admite, no mandado de segurança, a abertura de fase instrutória, já que a prova é toda pré-constituída - ou seja, no momento da propositura da ação -, o que torna o julgamento da causa mais rápido.

\footnotetext{
${ }^{12}$ MARQUES, S. B.; DALLARI, S. G. op. cit.

${ }^{13}$ SILVA, A. F. da; SILVA, K. A. da; VACCARO, S. B.; BARBOSA, F. M. Análise da judicialização da saúde pública no Espírito Santo. Rev. SJRJ, Rio de Janeiro, v. 19, n. 35, p. 75-90, dez. 2012.

${ }^{14}$ TRAVASSOS, D. V.; FERREIRA, R. C.; VARGAS, A. M. D.; MOURA, R. N. V. de; CONCEIÇÃO, E. M. de A; MARQUES, D. de F.; FERREIRA, E. F. e. Judicialização da saúde: um estudo de caso de três tribunais brasileiros. Ciência \& Saúde Coletiva, Rio de Janeiro, v. 18, n. 11, p. 3419-3429, nov. 2013. http://dx.doi. org/10.1590/S1413-81232013001100031.

${ }^{15}$ ROMERO, L. C. A jurisprudência do Tribunal de Justiça do Distrito Federal em ações de medicamentos. Revista de Direito Sanitário, São Paulo, v. 11, n. 2, p. 1-48, out. 2010. http://dx.doi.org/10.11606/issn.23169044.v11i2p11-59.

${ }^{16} \mathrm{CHIEFFI}$, A. L.; BARATA, R. B. Judicialização da política de assistência farmacêutica e equidade. Cad. Saúde Pública, Rio de Janeiro, v. 25, n. 8, p. 1843, ago. 2009. http://dx.doi.org/10.1590/ S0102-311X2009000800020.

${ }^{17}$ TRAVASSOS, D. V.; FERREIRA, R. C.; VARGAS, A. M. D.; MOURA, R. N. V. de; CONCEIÇÃO, E. M. de A; MARQUES, D. de F.; FERREIRA, E. F. e. op. cit.
} 


\section{Gênero do autor da ação}

As mulheres foram maioria como autoras das ações judiciais em São João da Boa Vista, resultado obtido também no Município de São Paulo, por Vieira e $Z_{u c c h i}{ }^{18}$; no Estado de Minas Gerais, por Machado et al..$^{19}$; e também no Estado de Minas Gerais, por Campos Neto et al. ${ }^{20}$. Uma possível explicação para o maior número de ações judiciais impetradas por mulheres seria a maior consciência delas em relação à saúde ${ }^{21}$.

\section{Representação judicial do autor da ação}

Em São João da Boa Vista, quanto à representação jurídica, há o predomínio de advogados particulares - em 49 das ações, significando 56,32\% -, sendo que as outras 38 ações foram patrocinadas através do convênio OAB/PGE. Também foi encontrada representação jurídica privada predominante nos estudos de Marques e Dallari $^{22}$, no Estado de São Paulo; de Vieira e Zucchi ${ }^{23}$, no Município de São Paulo; de Chieffi e Barata ${ }^{24}$, no Estado de São Paulo; de Pereira et al. ${ }^{25}$, no Estado de Santa Catarina; de Machado et al. ${ }^{26}$, no Estado de Minas Gerais; e de Campos Neto et al..$^{27}$, no Estado de Minas Gerais.

O fato de essas ações geralmente serem ajuizadas por advogados particulares mostra que os pacientes arcaram com os custos dessa representação e, em princípio, poderiam adquirir os medicamentos solicitados ${ }^{28}$, além de poderem, ainda, ter um maior conhecimento de seus direitos ${ }^{29}$.

\footnotetext{
${ }^{18}$ VIEIRA, F. S.; ZUCCHI, P. Distorções causadas pelas ações judiciais à política de medicamentos no Brasil. Revista de Saúde Pública. São Paulo, v. 41, n. 2, p. 214-222, abr. 2007. http://dx.doi.org/10.1590/S003489102007000200007.

${ }^{19}$ MACHADO, M. A. de A.; ACURCIO, F. de A.; BRANDÃO, C. M. R.; FALEIROS, D. R.; GUERRA JR, A. A.; CHERCHIGLIA, M. L.; ANDRADE, E. I. G. Judicialização do acesso a medicamentos no Estado de Minas Gerais, Brasil. Revista de Saúde Pública, São Paulo, v. 45, n. 3, p. 590-598, jun. 2011. http://dx.doi.org/10.1590/ S0034-89102011005000015.

${ }^{20}$ CAMPOS NETO, O. H.; ACURCIO, F. de A.; MACHADO, M. A. de A.; FERRÉ, F.; BARBOSA, F. L. V.; CHERCHIGLIA, M. L.; ANDRADE, E. I. G. Médicos, advogados e indústria farmacêutica na judicialização da saúde em Minas Gerais, Brasil. Revista de Saúde Pública, São Paulo, v. 46, n. 5, p. 784-790, out. 2012. http://dx.doi. org/10.1590/S0034-89102012000500004.

${ }^{21}$ VIEIRA, F. S.; ZUCCHI, P. op. cit.

${ }^{22}$ MARQUES, S. B.; DALLARI, S. G. op. cit.

${ }^{23}$ VIEIRA, F. S.; ZUCCHI, P. op. cit.

${ }^{24} \mathrm{CHIEFFI,} \mathrm{A.} \mathrm{L.;} \mathrm{BARATA,} \mathrm{R.} \mathrm{B.} \mathrm{op.} \mathrm{cit.}$

${ }^{25}$ PEREIRA, J. R.; SANTOS, R. I. dos; NASCIMENTO JUNIOR, J. M. do; SCHENKEL, E. P. Análise das demandas judiciais para o fornecimento de medicamentos pela Secretaria de Estado da Saúde de Santa Catarina nos anos de 2003 e 2004. Ciência \& Saúde Coletiva, Rio de Janeiro, v. 15, supl. 3, p. 3551-3560, 2010. http://dx.doi.org/10.1590/S1413-81232010000900030.

${ }^{26}$ MACHADO, M. A. de A.; ACURCIO, F. de A.; BRANDÃO, C. M. R.; FALEIROS, D. R.; GUERRA JR, A. A.; CHERCHIGLIA, M. L.; ANDRADE, E. I. G. op. cit.

${ }^{27}$ CAMPOS NETO, O. H.; ACURCIO, F. de A.; MACHADO, M. A. de A.; FERRÉ, F.; BARBOSA, F. L. V.; CHERCHIGLIA, M. L.; ANDRADE, E. I. G. op. cit.

${ }^{28} \mathrm{CHIEFFI,} \mathrm{A.} \mathrm{L.;} \mathrm{BARATA,} \mathrm{R.} \mathrm{B.} \mathrm{op.} \mathrm{cit.}$

${ }^{29}$ MACHADO, M. A. de A.; ACURCIO, F. de A.; BRANDÃO, C. M. R.; FALEIROS, D. R.; GUERRA JR, A. A.; CHERCHIGLIA, M. L.; ANDRADE, E. I. G. op. cit.
} 
No mesmo sentido, Vieira e Zucchi sugerem que provavelmente essas pessoas tenham condições financeiras suficientes para custear os honorários do profissional.

[...] 63\% dos impetrantes de ações judiciais residirem nas áreas do município com menor grau de exclusão social vai ao encontro do achado de que a maioria desses pacientes teve representação privada de seus processos. Também sugere que são indivíduos menos carentes de proteção social que estão movendo ações contra o poder público municipal e instala a dúvida sobre o descumprimento das ações de equidade propostas pelo SUS ${ }^{30}$.

No Estado do Rio de Janeiro, porém, Messeder et al. ${ }^{31}$ identificaram como condutor da ação a Defensoria Pública, com tendência de crescimento de sua participação no patrocínio das ações; Sant'Ana ${ }^{32}$ confirmou o achado de Messeder et al. ${ }^{33}$, pois a Defensoria Pública conduziu 70,4\% das ações no Estado do Rio de Janeiro; assim como observaram Travassos et al. ${ }^{34}$ nos Tribunais de Justiça dos estados de Minas Gerais, Rio Grande do Sul e Pernambuco uma maior representatividade da Defensoria Pública.

\section{Gratuidade de justiça}

No Município de São João da Boa Vista, a gratuidade de justiça foi concedida em 82 ações, representando 94,25\%. Dado muito parecido encontrou Sant'Ana ${ }^{35}$ no Estado do Rio de Janeiro, onde a gratuidade de justiça não foi concedida somente em uma única ação. No âmbito da pesquisa de Ventura et al. ${ }^{36}$, realizada no Tribunal de Justiça do Estado do Rio de Janeiro, em nenhum processo foi negada a gratuidade de justiça.

[...], no SUS, a gratuidade é pressuposto do acesso, enquanto que, no sistema da justiça, a gratuidade não é regra e somente será concedida se houver comprovação da necessidade econômica do autor, podendo inclusive ser impugnada pela parte contrária. Isto implica que o autor, para se beneficiar da gratuidade, deve comprovar sua impossibilidade de arcar com as despesas processuais da ação judicial, ou seja, sua hipossuficiência econômica, mas não necessariamente lhe é exigido demonstrar a impossibilidade de arcar com os custos do insumo e/ou do procedimento de saúde requeridos ${ }^{37}$. (Destaque das autoras)

\footnotetext{
${ }^{30}$ VIEIRA, F. S.; ZUCCHI, P. op. cit., p. 220.

${ }^{31}$ MESSEDER, A. M.; CASTRO, C. G. S. O. de; LUIZA, V. L. op. cit.

${ }^{32}$ SANT'ANA, J. M. B. op. cit.

${ }^{33}$ MESSEDER, A. M.; CASTRO, C. G. S. O. de; LUIZA, V. L. op. cit.

${ }^{34}$ TRAVASSOS, D. V.; FERREIRA, R. C.; VARGAS, A. M. D.; MOURA, R. N. V. de; CONCEIÇÃO, E. M. de A; MARQUES, D. de F.; FERREIRA, E. F. e. op. cit.

${ }^{35}$ SANT'ANA, J. M. B. op. cit.

${ }^{36}$ VENTURA, M.; SIMAS, L.; PEPE, V. L. E.; SCHRAMM. Judicialização da saúde, acesso à justiça e a efetividade do direito à saúde. Physis: Revista de Saúde Coletiva, Rio de Janeiro, v. 20, n. 1, p. 77-100, 2010. http:// dx.doi.org/10.1590/S0103-73312010000100006.

${ }^{37}$ Id. Ibid., p. 90.
} 
Ventura et al. ${ }^{38}$ constataram que a solicitação e o deferimento da gratuidade de justiça e, consequentemente, o reconhecimento judicial da hipossuficiência econômica do reivindicante, são uma característica majoritária da demanda judicial de medicamentos.

\section{Natureza do réu}

A diretora do Departamento Municipal de Saúde de São João da Boa Vista foi ré em $56,32 \%$ das ações. Marques e Dallari ${ }^{39}$ encontraram número semelhante no Estado de São Paulo, em que o Secretário de Estado da Saúde figurava como réu em $61 \%$ das ações. Porém, Silva et al.$^{40}$, no Espírito Santo, identificaram que todas as demandas eram contra a Fazenda Pública.

Uma explicação para acionar como ré a diretora do Departamento Municipal de Saúde de São João da Boa Vista é a própria escolha da ação, ou seja, o mandado de segurança exige como réu uma pessoa física que exerça autoridade; além do mais, a esfera municipal, das três esferas de governo, é a que está mais próxima do cidadão.

No Rio de Janeiro, Messeder et al. ${ }^{41}$ encontraram que 50,2\% das ações foram impetradas contra o estado e o município, sendo que apenas 35,5\% contra o estado; já Sant'Ana ${ }^{42}$, no Estado do Rio de Janeiro, observou apenas 25,9\% das ações tendo como réu o estado; no Distrito Federal, Romero ${ }^{43}$ identificou que 70,7\% das ações tinham como réu o Distrito Federal e que, em 23,5\%, o réu era o secretário da Saúde. Destaque-se que, em alguns casos, as ações são propostas não só contra o estado, mas também contra o município onde reside o autor da ação ${ }^{44}$.

\section{Resultado da sentença}

A maioria das ações solicitando medicamentos em São João da Boa Vista foi julgada procedente, representando 93,1\%. Marques e Dallari ${ }^{45}$, no Estado de São Paulo, encontraram 90,3\% das ações julgadas procedentes; Borges e Ugá ${ }^{46}$ também chegaram a um número semelhante no Estado do Rio de Janeiro, onde $89 \%$ das ações foram julgadas procedentes; Romero ${ }^{47}$, no Distrito Federal, apontou que todos

\footnotetext{
${ }^{38}$ VENTURA, M.; SIMAS, L.; PEPE, V. L. E.; SCHRAMM. op. cit. p. 90.

${ }^{39}$ MARQUES, S. B.; DALLARI, S. G. op. cit.

${ }^{40}$ SILVA, A. F. da; SILVA, K. A. da; VACCARO, S. B.; BARBOSA, F. M. op. cit.

${ }^{41}$ MESSEDER, A. M.; CASTRO, C. G. S. O. de; LUIZA, V. L. op. cit.

${ }^{42}$ SANT'ANA, J. M. B. op. cit.

${ }^{43}$ ROMERO, L. C. op. cit.

${ }^{44}$ BORGES, D. da C. L.; UGÁ, M. A. D. Conflitos e impasses da judicialização na obtenção de medicamentos: as decisões de $1^{\mathrm{a}}$ instância nas ações individuais contra o Estado do Rio de Janeiro, Brasil, em 2005. Cad. Saúde Pública, Rio de Janeiro, v. 26, n. 1, p. 59-69, jan. 2010. http://dx.doi.org/10.1590/S0102-311X2010000100007.

${ }^{45}$ MARQUES, S. B.; DALLARI, S. G. op. cit.

${ }^{46}$ BORGES, D. da C. L.; UGÁ, M. A. D. op. cit.

${ }^{47}$ ROMERO, L. C. op. cit.
} 
os pleitos foram concedidos; Travassos et al. ${ }^{48}$, nos Tribunais dos estados de Minas Gerais, Rio Grande do Sul e Pernambuco, identificaram que a maioria das decisões foi favorável aos usuários (97,8\%); e Silva et al. ${ }^{49}$, no Tribunal de Justiça do Espírito Santo, obtiveram resultados que evidenciaram a majoritária procedência das ações.

Isso indica que o Poder Judiciário tem se manifestado sempre em favor do usuário quando se trata de solicitações sobre medicamentos, independentemente de ser aquele medicamento padronizado pelo Ministério da Saúde ${ }^{50}$. Há uma constatação comum nos estudos de que a prescrição médica é o principal respaldo para a decisão do juiz ${ }^{51}$.

\section{Origem da prescrição}

A receita médica proveniente do serviço privado foi maioria, pois originou $70,11 \%$ das ações interpostas em face do Município de São João da Boa Vista. Pereira et $a l .{ }^{52}$, no Estado de Santa Catarina, observaram que a prescrição anexada era proveniente de serviços privados de saúde (55,8\%); Machado et al. ${ }^{53}$ encontraram, no Estado de Minas Gerais, um número próximo ao desta pesquisa, ou seja, 70,5\% dos autores foram atendidos no sistema privado de saúde; Campos Neto et al ${ }^{54}$ identificaram, em Minas Gerais, predomínio de atendimento por médicos do setor privado $(87,5 \%)$. Esses dados demonstram que a população com maior poder aquisitivo é que está se beneficiando do resultado das ações judiciais ${ }^{55}$.

Em razão disso, as demandas judiciais estão ferindo o princípio da equidade do SUS, isto é, as ações judiciais não estão fornecendo medicamentos a quem utiliza o sistema público de saúde e depende do fornecimento gratuito de medicamentos ${ }^{56}$.

Pereira et al..$^{57}$ observaram um predomínio de prescrições originárias no serviço privado, o que os levou a afirmar a existência de um público misto, capaz de custear consultas e eventuais exames, diretamente ou por meio da medicina suplementar, mas que recorre ao SUS para a obtenção dos medicamentos.

\footnotetext{
${ }^{48}$ TRAVASSOS, D. V.; FERREIRA, R. C.; VARGAS, A. M. D.; MOURA, R. N. V. de; CONCEIÇÃO, E. M. de A; MARQUES, D. de F.; FERREIRA, E. F. e. op. cit.

${ }^{49}$ SILVA, A. F. da; SILVA, K. A. da; VACCARO, S. B.; BARBOSA, F. M. op. cit.

${ }^{50}$ BORGES, D. da C. L.; UGÁ, M. A. D. op. cit.

${ }^{51}$ PEPE, V. L. E.; FIGUEIREDO, T. de A.; SIMAS, L.; CASTRO, C. G. S. O. de; VENTURA, M. A judicialização da saúde e os novos desafios da gestão da assistência farmacêutica. Ciência \& Saúde Coletiva, Rio de Janeiro, v. 15, n. 5, p. 2405-2411, ago. 2010. http://dx.doi.org/10.1590/S1413-81232010000500015.

${ }^{52}$ PEREIRA, J. R.; SANTOS, R. I. dos; NASCIMENTO JUNIOR, J. M. do; SCHENKEL, E. P. op. cit.

${ }^{53}$ MACHADO, M. A. de A.; ACURCIO, F. de A.; BRANDÃO, C. M. R.; FALEIROS, D. R.; GUERRA JR, A. A.; CHERCHIGLIA, M. L.; ANDRADE, E. I. G. op. cit.

${ }^{54}$ CAMPOS NETO, O. H.; ACURCIO, F. de A.; MACHADO, M. A. de A.; FERRÉ, F.; BARBOSA, F. L. V.; CHERCHIGLIA, M. L.; ANDRADE, E. I. G. op. cit.

${ }^{55}$ CHIEFFI, A. L.; BARATA, R. B. op. cit.

${ }^{56}$ Id. Ibid.

${ }^{57}$ PEREIRA, J. R.; SANTOS, R. I. dos; NASCIMENTO JUNIOR, J. M. do; SCHENKEL, E. P. op. cit.
} 
Em São João da Boa Vista, as receitas médicas encartadas nos autos continham vários remédios solicitados, sendo eles tanto disponibilizados pelos programas de assistência farmacêutica do SUS, como também medicamentos não contemplados pela lista do SUS. Porém, como o Departamento Municipal de Saúde de São João da Boa Vista somente poderia fornecer medicamento se o usuário se enquadrasse no artigo 28 do Decreto n. 7.508/2011 ${ }^{58}$ - ou seja, assistido pelo SUS, com receita proveniente do SUS durante atendimento em unidade de saúde do SUS e remédio solicitado genérico na lista do SUS -, muitas das receitas acabaram por desembocar no Poder Judiciário.

\section{Competência para fornecimento de medicamentos}

Com relação à competência para fornecimento dos medicamentos solicitados judicialmente em São João da Boa Vista, 56,39\% não pertenciam à lista oficial; 30,40\% eram de responsabilidade do município e 13,21\% de responsabilidade do estado.

Portanto, a maioria dos medicamentos pedidos nas ações judiciais pelo sanjoanense não era padronizada pelo SUS. Chieffi e Barata ${ }^{59}$ encontraram, no Estado de São Paulo, que 77\% dos medicamentos não pertenciam aos programas de assistência farmacêutica do SUS; Pereira et al. ${ }^{60}$, em Santa Catarina, identificaram 62,2\% dos medicamentos como não padronizados; Machado et al. ${ }^{61}$, constataram, em Minas Gerais, que aproximadamente 56\% dos medicamentos não estavam incluídos em programas do SUS, resultado semelhante ao encontrado nesta pesquisa; Silva et al. ${ }^{62}$, no Espírito Santo, apontaram que o maior pleito foi em relação aos medicamentos não padronizados.

Para Sant'Ana ${ }^{63}$, é razoável supor que, em geral, os medicamentos não pertencentes às listas são os principais motivadores das demandas judiciais. Esses dados sugerem que a população tem acesso aos medicamentos referentes aos programas de fornecimento do SUS e que não tem ocorrido descontinuidade de abastecimento, pois a maioria das solicitações se liga a medicamentos não incluídos nos programas ${ }^{64}$.

Vieira e Zucchi ${ }^{65}$, no Município de São Paulo, constataram que 62\% dos itens solicitados fazem parte de listas de medicamentos de programas do SUS;

\footnotetext{
${ }^{58}$ BRASIL. Decreto n. 7.508 de 28 de junho de 2011. Regulamenta a Lei $n^{\circ} 8.080$, de 19 de setembro de 1990, para dispor sobre a organização do Sistema Único de Saúde - SUS, o planejamento da saúde, a assistência à saúde e a articulação interfederativa, e dá outras providências. Disponível em: <http://www. planalto.gov.br/ccivil_03/_ato2011-2014/2011/decreto//D7508.htm>. Acesso em: 16 fev. 2014.

${ }^{59} \mathrm{CHIEFFI,} \mathrm{A.} \mathrm{L.;} \mathrm{BARATA,} \mathrm{R.} \mathrm{B.} \mathrm{op.} \mathrm{cit.}$

${ }^{60}$ PEREIRA, J. R.; SANTOS, R. I. dos; NASCIMENTO JUNIOR, J. M. do; SCHENKEL, E. P. op. cit.

${ }^{61}$ MACHADO, M. A. de A.; ACURCIO, F. de A.; BRANDÃO, C. M. R.; FALEIROS, D. R.; GUERRA JR, A. A.; CHERCHIGLIA, M. L.; ANDRADE, E. I. G. op. cit.

${ }^{62}$ SILVA, A. F. da; SILVA, K. A. da; VACCARO, S. B.; BARBOSA, F. M. op. cit.

${ }^{63}$ SANT'ANA, J. M. B. op. cit.

${ }^{64}$ CHIEFFI, A. L.; BARATA, R. B. op. cit.

${ }^{65}$ VIEIRA, F. S.; ZUCCHI, P. op. cit.
} 
Sant'Ana ${ }^{66}$, no Estado do Rio de Janeiro, observou 57,4\% dos medicamentos solicitados compõem a lista do SUS; Borges e Ugá ${ }^{67}$, também no Estado do Rio de Janeiro, identificaram que $52 \%$ dos medicamentos pleiteados estão padronizados pelo Ministério da Saúde; Romero ${ }^{68}$, no Distrito Federal, identificou que 47,6\% dos medicamentos solicitados faziam parte da lista oficial; Campos Neto et al. ${ }^{69}$, em Minas Gerais, encontraram que a maioria dos medicamentos solicitados era fornecida pelas políticas de assistência farmacêutica do SUS.

\begin{abstract}
Os mandados judiciais podem interferir tanto nas questões orçamentárias como administrativas, ao determinarem a entrega de medicamentos que não são de responsabilidade do ente federativo, réu na ação judicial, conforme as pactuações existentes, ou mesmo quando de responsabilidade do réu, mas não programados pelos serviços ${ }^{70}$.
\end{abstract}

\title{
10. Registro do medicamento na Anvisa
}

Todos os medicamentos solicitados judicialmente no período estudado em São João da Boa Vista possuíam registro na Anvisa, dado também encontrado por Vieira e $Z u c c h{ }^{71}$ no Município de São Paulo.

É necessário salientar que, no Município de São João da Boa Vista, não há hospital universitário, o que poderia explicar a inexistência de solicitação de medicamentos sem registro na Anvisa, já que são os hospitais universitários a prescrever fármacos inovadores, em razão de usarem rotineiramente novas tecnologias e estudos clínicos ${ }^{72,73,74}$.

Marques e Dallari ${ }^{75}$, no Estado de São Paulo, descobriram que em 9,6\% dos casos analisados os medicamentos não possuíam registro na Anvisa; Sant'Ana ${ }^{76}$, no Estado do Rio de Janeiro, identificou 2,6\% dos medicamentos solicitados sem registro na agência; Pereira et al. ${ }^{77}$, em Santa Catarina, observaram determina-

\footnotetext{
${ }^{66}$ SANT'ANA, J. M. B. op. cit.

${ }^{67}$ BORGES, D. da C. L.; UGÁ, M. A. D. op. cit.

${ }^{68}$ ROMERO, L. C. op. cit.

${ }^{69}$ CAMPOS NETO, O. H.; ACURCIO, F. de A.; MACHADO, M. A. de A.; FERRÉ, F.; BARBOSA, F. L. V.; CHERCHIGLIA, M. L.; ANDRADE, E. I. G. op. cit.

${ }^{70}$ PEPE, V. L. E.; FIGUEIREDO, T. de A.; SIMAS, L.; CASTRO, C. G. S. O. de; VENTURA, M. op. cit.p. 2408.

${ }^{71}$ VIEIRA, F. S.; ZUCCHI, P. op. cit.

${ }^{72}$ MESSEDER, A. M.; CASTRO, C. G. S. O. de; LUIZA, V. L. op. cit.

${ }^{73}$ ROMERO, L. C. Judicialização das políticas de assistência farmacêutica: o caso do Distrito Federal. Consultoria Legislativa do Senado Federal. Textos para discussão 41, Brasília, maio 2008. Disponível em: <http:// www12.senado.gov.br/publicacoes/estudos-legislativos/tipos-de-estudos/textos-para-discussão/td-41-judicializacao-das-politicas-de-assistencia-farmaceutica-o-caso-do-distrito-federal>. Acesso em: 10 mar. 2014.

${ }^{74}$ PEPE, V. L. E.; FIGUEIREDO, T. de A.; SIMAS, L.; CASTRO, C. G. S. O. de; VENTURA, M. op. cit.

${ }^{75}$ MARQUES, S. B.; DALLARI, S. G. op. cit.

${ }^{76}$ SANT'ANA, J. M. B. op. cit.

${ }^{77}$ PEREIRA, J. R.; SANTOS, R. I. dos; NASCIMENTO JUNIOR, J. M. do; SCHENKEL, E. P. op. cit.
} 
ções judiciais obrigando o fornecimento pelo estado de medicamentos de uso off label, além de medicamentos que ainda não tinham seu registro na Anvisa (1,4\%), havendo inclusive medicamentos importados; Machado et al.$^{78}$, em Minas Gerais, identificaram $4,8 \%$ dos medicamentos pleiteados como não registrados na agência.

\section{Uso do nome comercial na prescrição dos medicamentos}

Na pesquisa realizada em São João da Boa Vista, chamou atenção também o fato de a maioria das receitas constantes nos autos prescrever medicamentos pelo nome comercial, não obstante a Lei Federal n. 9.787/199979 e a Lei Municipal n. $573 / 2000^{80}$ obrigarem a descrição do medicamento pelo nome genérico na rede pública. Dado também encontrado por Borges e Ugá ${ }^{81}$, no Estado do Rio de Janeiro, em que muitos dos medicamentos estavam descritos por seus nomes comerciais; e por Marques e Dallari ${ }^{82}$, no Estado de São Paulo, em que 77,4\% dos autores requereram medicamentos de determinada marca.

Contrariamente, no Distrito Federal, Romero ${ }^{83}$ encontrou 58,5\% dos medicamentos solicitados descritos pelo nome genérico, ao passo que $40,1 \%$ eram prescritos pelo nome de marca.

Ventura et al. ${ }^{84}$ observaram que a demanda judicial evidenciou uma tensão interna no sistema de assistência à saúde, entre a autonomia do médico (mesmo se funcionário do sistema público de saúde) em sua prescrição à pessoa atendida e os regulamentos e as normas sanitárias.

\section{Gastos com ações judiciais}

Em São João da Boa Vista, os gastos com as ações judiciais quase triplicaram de 2010 para 2012, sendo que o gasto médio por ação teve um salto de aproximadamente $500 \%$ nesse período. Em Santa Catarina, o gasto médio por ação obteve um incremento de $326 \%$ dos anos 2001/2002 para os anos 2003/2004 $4^{85}$.

\footnotetext{
${ }^{78}$ MACHADO, M. A. de A.; ACURCIO, F. de A.; BRANDÃO, C. M. R.; FALEIROS, D. R.; GUERRA JR, A. A.; CHERCHIGLIA, M. L.; ANDRADE, E. I. G. op. cit.

${ }^{79}$ BRASIL. Lei n. 9.787 de 10 de fevereiro de 1999. Altera a Lei $n^{\circ}$ 6.360, de 23 de setembro de 1976, que dispõe sobre a vigilância sanitária, estabelece o medicamento genérico, dispõe sobre a utilização de nomes genéricos em produtos farmacêuticos e dá outras providências. Disponível em: <http://www. planalto. gov.br/ccivil_03/leis/19787.htm>. Acesso em: 11 mar. 2014.

${ }^{80}$ CÂMARA MŪNICIPAL DE SÃO JOÃO DA BOA VISTA. Lei n. 573 de 10 de novembro de 2000. Dispõe sobre a obrigatoriedade da aquisição de remédios genéricos pela rede pública municipal de saúde e exige que os médicos da mesma rede receitem medicamentos sempre através de seus nomes genéricos. Disponível em: <http://www.digitechsistemas.com.br/saojoaodaboavista/Default.aspx>. Acesso em: 16 fev. 2014.

${ }^{81}$ BORGES, D. da C. L.; UGÁ, M. A. D. op. cit.

${ }^{82}$ MARQUES, S. B.; DALLARI, S. G. op. cit.

${ }^{83}$ ROMERO, L. C. A jurisprudência do Tribunal de Justiça do Distrito Federal em ações de medicamentos, cit.

${ }^{84}$ VENTURA, M.; SIMAS, L.; PEPE, V. L. E.; SCHRAMM. op. cit.

${ }^{85}$ PEREIRA, J. R.; SANTOS, R. I. dos; NASCIMENTO JUNIOR, J. M. do; SCHENKEL, E. P. op. cit.
} 
No Município de São João da Boa Vista, os gastos com ações judiciais representaram 18,76\% da assistência farmacêutica em 2010; em 2012, essa proporção quase dobrou, chegando a 30,20\%, representando um terço dos gastos com a assistência farmacêutica. Índices menores foram encontrados em Santa Catarina por Pereira et al. ${ }^{86}$ : as demandas judiciais foram responsáveis, em 2003, por aproximadamente $7,5 \%$ e em 2004, por aproximadamente $11,5 \%$ do que foi gasto com remédios pela Secretaria Estadual da Saúde.

Essas ações têm consequências orçamentárias importantes, uma vez que os recursos são finitos e sua administração deve ser planejada e balizada pelas políticas de saúde ${ }^{87}$. O cumprimento de determinações judiciais para fornecimento de medicamentos, insumos e serviços de saúde acarreta gastos elevados e não programados ${ }^{88}$. O processo judicial força o deslocamento de recursos públicos das políticas de saúde para o atendimento das demandas individualizadas, ou seja, busca-se uma solução individualizada para uma questão coletiva ${ }^{89}$.

$\mathrm{O}$ crescimento do número de ações judiciais, associado ao fato de que as decisões são predominantemente favoráveis aos autores, vem gerando problemas para o sistema de saúde como um todo, já que algumas decisões acabam comprometendo gravemente o orçamento da saúde em razão do elevado preço de alguns medicamentos pleiteados - que, em muitos casos, não se encontram nos elencos oficiais da regulamentação da assistência farmacêutica no âmbito do SUS ${ }^{90}$.

Se, por um lado, as decisões judiciais visam a garantir o atendimento ao direito e às necessidades do indivíduo, por outro, remetem ao gestor a responsabilidade de decisões administrativas que muitas vezes contradizem os princípios constitucionais da equidade e do acesso à assistência à saúde de qualidade ${ }^{91}$.

\section{Considerações finais}

A Recomendação n. 31/2010 do CNJ' - embora tardiamente, posto que elaborada somente após 11 anos do início das muitas ações judiciais solicitando

\footnotetext{
${ }^{86}$ PEREIRA, J. R.; SANTOS, R. I. dos; NASCIMENTO JUNIOR, J. M. do; SCHENKEL, E. P. op. cit.

${ }^{87} \mathrm{CHIEFFI,} \mathrm{A.} \mathrm{L.;} \mathrm{BARATA,} \mathrm{R.} \mathrm{B.} \mathrm{op.} \mathrm{cit.}$

${ }^{88}$ MACHADO, M. A. de A.; ACURCIO, F. de A.; BRANDÃO, C. M. R.; FALEIROS, D. R.; GUERRA JR, A. A.; CHERCHIGLIA, M. L.; ANDRADE, E. I. G. op. cit.

${ }^{89} \mathrm{CHIEFFI,} \mathrm{A.} \mathrm{L.;} \mathrm{BARATA,} \mathrm{R.} \mathrm{B.} \mathrm{op.} \mathrm{cit.}$

${ }^{90}$ BORGES, D. da C. L.; UGÁ, M. A. D. op. cit.

${ }^{91}$ ANDRADE, E. I. G.; MACHADO, C. D.; FALEIROS, D. R.; SZUSTER, D. A. C.; GUERRA JÚNIOR, A. A.; SILVA, G. D. da; CHERCHIGLIA; ACÚRCIO, F. de A. A judicialização da saúde e a política nacional de assistência farmacêutica no Brasil: gestão da clínica e medicalização da justiça. Revista Médica de Minas Gerais, Belo Horizonte, v. 18, n. 4, supl. 4, p. 46-50, 2008.

${ }^{92}$ CONSELHO NACIONAL DE JUSTIÇA. Recomendação n. 31 de 30 de março de 2010. Recomenda aos Tribunais a adoção de medidas visando subsidiar os magistrados e demais operadores do direito, para assegurar maior eficiência na solução das demandas judiciais envolvendo a assistência à saúde. Disponível em: <http://www.cnj.jus.br/atos-administrativos/atos-da-presidencia/322-recomendacoes-do-conselho/12113-recomendacao-no-30-de-marco-de-2010?tmpl=component\&print=I\&Page=>. Acesso em: 17 maio 2012.
} 
medicamentos -, representou um avanço na área e um significativo enfrentamento da questão, visto que, entre outras resoluções, recomendou aos tribunais de justiça estaduais que:

a) até dezembro de 2010 celebrem convênios que objetivem disponibilizar apoio técnico composto por médicos e farmacêuticos para auxiliar os magistrados na formação de um juízo de valor quanto à apreciação das questões clínicas apresentadas pelas partes das ações relativas à saúde, observadas as peculiaridades regionais $(. . .)^{93}$.

No Estado de São Paulo, com atraso de quase dois anos em relação ao prazo estipulado pela Recomendação n. 31, o Termo de Cooperação Técnica, assinado em 3 de setembro de 2012, teve como objetivo disponibilizar aos magistrados apoio e informações técnicas nas áreas de saúde e de Direito Sanitário ${ }^{94}$.

Entretanto, entende-se ser este Termo de Cooperação Técnica um imprescindível instrumento para os magistrados, pois assim eles têm à sua disposição, de forma rápida, um instrumento técnico direcionado especificamente à judicialização da saúde.

Apesar da existência da recomendação do CNJ e também do Termo de Cooperação Técnica no período pesquisado, foi observado em São João da Boa Vista um número crescente de ações judiciais para acesso a medicamentos, com significativo impacto no orçamento da saúde municipal e prejuízo para a coletividade, em função do atendimento ao direito individual, posto que não se levaram em consideração os limites orçamentários estatais.

Quanto à gestão pública, sugere-se realizar uma melhor racionalização do uso dos recursos públicos, com o planejamento de ações em saúde para toda a população, e não somente açães emergenciais, como é o caso das demandas judiciais.

Com relação aos juízes, sugere-se a solicitação de apoio técnico para embasar suas decisões em relação a medicamentos, ou seja, realmente utilizarem, no caso do Estado de São Paulo, o Termo de Cooperação Técnica firmado entre o governo do estado, o tribunal de justiça e a Escola Paulista da Magistratura, direcionado à judicialização da saúde, a fim de disponibilizar aos juízes um banco de dados na área da saúde ${ }^{95}$.

Sugere-se, também, mais conversa e intercâmbio entre os poderes Executivo e Judiciário, a fim de que este conheça a política pública na área da saúde e aquele envie informações técnicas atualizadas, para que os magistrados possam decidir realizando justiça social.

\footnotetext{
${ }^{93}$ CONSELHO NACIONAL DE JUSTIÇA, cit. p. 2.

${ }^{94}$ TRIBUNAL DE JUSTIÇA DO ESTADO DE SÃO PAULO. Termo de cooperação técnica. São Paulo, set. 2012. Disponível em: <www.tjsp.jus.br/09-03-12_termo_saude[1].pdf>. Acesso em: 20 fev. 2014.

${ }^{95}$ Id. Ibid.
} 
Sugere-se, ainda, outra forma de solução desse tipo de conflito, saindo da esfera contenciosa e ingressando na esfera administrativa, através da mediação ${ }^{96}$. Poder-se-ia criar, no âmbito municipal, uma assessoria municipal de mediação na área da saúde, com objetivo de resolução administrativa de conflitos envolvendo concessão de procedimentos de saúde, especialmente questões sobre fornecimento de medicamentos. Isso diminuiria os gastos tanto do ente público como do solicitante, em virtude dos custos de uma demanda judicial.

Como observaram Pereira et al. ${ }^{97}$, torna-se necessária uma melhor organização do Estado no tocante à assistência farmacêutica, especialmente diante do crescimento do número de ações judiciais solicitando medicamentos, bem como uma melhor alocação dos recursos disponíveis, a fim de não comprometer o direito constitucional à saúde dos indivíduos.

\section{Referências}

ANDRADE, E. I. G.; MACHADO, C. D.; FALEIROS, D. R.; SZUSTER, D. A. C.; GUERRA JÚNIOR, A. A.; SILVA, G. D. da; CHERCHIGLIA; ACÚRCIO, F. de A. A judicialização da saúde e a política nacional de assistência farmacêutica no Brasil: gestão da clínica e medicalização da justiça. Revista Médica de Minas Gerais, Belo Horizonte, v. 18, n. 4, supl. 4, p. 46-50, 2008.

BARROSO, L. R. Da falta de efetividade à judicialização excessiva: direito à saúde, fornecimento gratuito de medicamentos e parâmetros para a atuação judicial. Rio de Janeiro, 2007. Disponível em: <http://s.conjur.com.br/dl/estudobarroso.pdf>. Acesso em: 22 out. 2011.

BORGES, D. da C. L.; UGÁ, M. A. D. Conflitos e impasses da judicialização na obtenção de medicamentos: as decisões de $1^{\text {a }}$ instância nas ações individuais contra o Estado do Rio de Janeiro, Brasil, em 2005. Cad. Saúde Pública, Rio de Janeiro, v. 26, n. 1, p. 59-69, jan. 2010. http://dx.doi.org/10.1590/S0102-311X2010000100007.

BRANCO, L. T. C. Abrangência do direitoà saúde: fornecimento de medicamentos especiaisé dever do Estado? Revista do Movimento do Ministério Público Democrático, São Paulo, ano 4, n. 13, maio 2007.

CAMPOS NETO, O. H.; ACURCIO, F. de A.; MACHADO, M. A. de A.; FERRÉ, F.; BARBOSA, F. L. V.; CHERCHIGLIA, M. L.; ANDRADE, E. I. G. Médicos, advogados e indústria farmacêutica na judicialização da saúde em Minas Gerais, Brasil. Revista de Saúde Pública, São Paulo, v. 46, n. 5, p. 784-790, out. 2012. http://dx.doi.org/10.1590/S0034-89102012000500004.

CHIEFFI, A. L.; BARATA, R. B. Judicialização da política de assistência farmacêutica e equidade. Cad. Saúde Pública, Rio de Janeiro, v. 25, n. 8, p. 1839-1849, ago. 2009. http://dx.doi.org/10.1590/S0102-311X2009000800020.

\footnotetext{
96 “É uma forma de negociação direta entre as partes em conflito em que terceira pessoa previamente treinada em técnicas de negociação, munida de conhecimentos multidisciplinares de psicologia e sociologia, é convidada não para decidir, mas para conduzir as partes a uma solução, facilitando o acordo". In: DINIZ, M. H. Dicionário jurídico universitário. São Paulo: Saraiva, 2010. p. 392..

${ }^{97}$ PEREIRA, J. R.; SANTOS, R. I. dos; NASCIMENTO JUNIOR, J. M. do; SCHENKEL, E. P. op. cit.
} 
DINIZ, M. H. Dicionário jurídico universitário. São Paulo: Saraiva, 2010.

FIRJAN. Índice FIRJAN de desenvolvimento municipal. Disponível em: $<$ http://www.firjan. org.br/ifdm/consulta-ao-indice/consulta-ao-indice-grafico.htm?UF=SP\&IdCidade $=354910$ \&Indicator-4\&Ano=2010>. Acesso em 16 jan. 2014.

IBGE.Cidades@. Disponível em: <http://cidades.ibge.gov.br/painel/painel. php? codmun=354910>. Acesso em: 16 fev. 2014.

MACHADO, M. A. de A.; ACURCIO, F. de A.; BRANDÃO, C. M. R.; FALEIROS, D. R.; GUERRA JR, A. A.; CHERCHIGLIA, M. L.; ANDRADE, E. I. G. Judicialização do acesso a medicamentos no Estado de Minas Gerais, Brasil. Revista de Saúde Pública, São Paulo, v. 45, n. 3, p. 590-598, jun. 2011. http://dx.doi.org/10.1590/S0034-89102011005000015.

MARQUES, S. B.; DALLARI, S. G. Garantia do direito social à assistência farmacêutica no Estado de São Paulo. Revista de Saúde Pública, São Paulo, v. 41, n. 1, p. 101-107, fev. 2007. http://dx.doi.org/10.1590/S0034-89102007000100014.

MESSEDER, A. M.; CASTRO, C. G. S. O. de; LUIZA, V. L. Mandados judiciais como ferramenta para garantia do acesso a medicamentos no setor público: a experiência do Estado do Rio de Janeiro, Brasil. Cad. Saúde Pública, Rio de Janeiro, v. 21, n. 2, p. 525-534, mar./abr. 2005. http://dx.doi.org/10.1590/S0102-311X2005000200019.

PEPE, V. L. E.; FIGUEIREDO, T. de A.; SIMAS, L.; CASTRO, C. G. S. O. de; VENTURA, M. A judicialização da saúde e os novos desafios da gestão da assistência farmacêutica. Ciência \& Saúde Coletiva, Rio de Janeiro, v. 15, n. 5, p. 2405-2411, ago. 2010. http://dx.doi.org/10.1590/S1413-81232010000500015.

PEREIRA, J. R.; SANTOS, R. I. dos; NASCIMENTO JUNIOR, J. M. do; SCHENKEL, E. P. Análise das demandas judiciais para o fornecimento de medicamentos pela Secretaria de Estado da Saúde de Santa Catarina nos anos de 2003 e 2004. Ciência \& Saúde Coletiva, Rio de Janeiro, v. 15, supl. 3, p. 3551-3560, 2010. http://dx.doi.org/10.1590/S1413-81232010000900030.

ROMERO, L. C. A jurisprudência do Tribunal de Justiça do Distrito Federal em ações de medicamentos. Revista de Direito Sanitário, São Paulo, v. 11, n. 2, p. 1-48, out. 2010. http:// dx.doi.org/10.11606/issn.2316-9044.v11i2p11-59.

Judicialização das políticas de assistência farmacêutica: o caso do Distrito Federal. Consultoria Legislativa do Senado Federal. Textos para discussão 41, Brasília, maio 2008. Disponível em: <http://www12.senado.gov.br/publicacoes/estudos-legislativos/ tipos-de-estudos/textos-para-discussão/td-41-judicializacao-das-politicas-de-assistenciafarmaceutica-o-caso-do-distrito-federal>. Acesso em: 10 mar. 2014.

SANT'ANA, J.M. B. Essencialidade e assistência farmacêutica: um estudo exploratório das demandas judiciais individuais para acesso a medicamentos no Estado do Rio de Janeiro. 2009. 94p. Dissertação (Mestrado) - Escola Nacional de Saúde Pública Sergio Arouca, ENSP, Rio de Janeiro, 2009.

SILVA, A. F. da; SILVA, K. A. da; VACCARO, S. B.; BARBOSA, F. M. Análise da judicialização da saúde pública no Espírito Santo. Rev. SJRJ, Rio de Janeiro, v. 19, n. 35, p. 75-90, dez. 2012. 
TRAVASSOS, D. V.; FERREIRA, R. C.; VARGAS, A. M. D.; MOURA, R. N. V. de; CONCEIÇÃO, E. M. de A; MARQUES, D. de F.; FERREIRA, E. F. e. Judicialização da saúde: um estudo de caso de três tribunais brasileiros. Ciência \& Saúde Coletiva, Rio de Janeiro, v. 18, n. 11, p. 3419-3429, nov. 2013. http://dx.doi.org/10.1590/S1413-81232013001100031.

TRIBUNAL DE JUSTIÇA DO ESTADO DE SÃO PAULO. Termo de cooperação técnica. São Paulo, set. 2012. Disponível em: <www.tjsp.jus.br/09-03-12_termo_saude[1].pdf >. Acesso em: 20 fev. 2014.

VENTURA, M.; SIMAS, L.; PEPE, V. L. E.; SCHRAMM. Judicialização da saúde, acesso à justiça e a efetividade do direito à saúde. Physis: Revista de Saúde Coletiva, Rio de Janeiro, v. 20, n. 1, p. 77-100, 2010. http://dx.doi.org/10.1590/S0103-73312010000100006.

VIEIRA, F. S. Ações judiciais e direito à saúde: reflexão sobre a observância aos princípios do SUS. Revista de Saúde Pública. São Paulo, v. 42, n. 2, 365-369, fev. 2008. http://dx.doi.org/10.1590/S0034-89102008005000010.

; ZUCCHI, P. Distorções causadas pelas ações judiciais à política de medicamentos no Brasil. Revista de Saúde Pública. São Paulo, v. 41, n. 2, p. 214-222, abr. 2007. http://dx.doi.org/10.1590/S0034-89102007000200007.

Ildelisa Cabral - Mestre em Desenvolvimento Sustentável e Qualidade de Vida pelo Centro Universitário das Faculdades Associadas de Ensino. Professora no Centro Universitário da Fundação de Ensino Octávio Bastos. Advogada. São João da Boa Vista/SP, Brasil. E-mail: ildelisa@adv.oabsp.org.br.

Laura Ferreira de Rezende - Pós-Doutora pelo Departamento de Ginecologia, Obstetrícia e Mastologia da Universidade Estadual Paulista "Júlio de Mesquita Filho". Professora no Centro Universitário das Faculdades Associadas de Ensino. Fisioterapeuta. São João da Boa Vista/SP, Brasil. 